Albrecht von Hallers (G. RudoLPH) über eine kurze Darstellung der Neuropathologie am Ausgang des 18. Jahrhunderts (G. RAтH) werden die Anfänge der Elektrophysiologie im U̇berblick geboten (D. Hüfmeier von Hagen). Die schöne Darstellung von Johannes Müllers Nervenphysiologie (J.STEudeL) führt über zur Bestimmung der Leitungsgeschwindigkeit im Nerven durch Hermann von Helmholtz (W.Blasius). DuBois-Reymonds große Verdienste um die Grundlegung der Elektrophysiologie werden durch Rотнsснин klar geschildert. Weitere Vorträge befassen sich mit A.Bezold (R.Herrlinger), Ludwig Türck (E.Lesky), Prohaska und Purkyně (A. Kruta), mit der Reflexphysiologie des 19. Jahrhunderts (G.GangGullhem), mit Carl Ludwig, dem universalen Lehrmeister der Physiologie (H.SchröEr). Es folgen Beiträge von Schweizer Ärzten zur Neuronentheorie: R.A.Koelliker, W.His, A.H.Forel (H.Buess) und zur Geschichte der Großhirnhistologie (Glees). Den Beschluß des inhaltsreichen Bandes bildet die Geschichte der Elektroencephalographie mit H. Berger (H. Fischgold). Damit ist ein besonders fruchtbarer Abschnitt der Wissenschaftsgeschichte auf dem Gebiet der Neurophysiologie unter kundiger Führung Roтнschuнs zur Darstelling gelangt, der in vielem auf die moderne Neurophysiologie vorausweist. Das inhaltsreiche Buch bildet ein kleines Kompendium der Geschichte der Neurophysiologie, das jedem historisch interessierten Physiologen und Arzt zur Lektüre empfohlen werden kann.

H. Fischer

\title{
8. Norman Dain, Concepts of Insanity in the U.S.A. 1789-1865. Rutgers Uni- versity Press 1964 (N.J.).
}

In diesem Buche möchte der Autor die Faktoren darstellen, die in Amerika der Überwindung einer «unwissenschaftlichen und unnötig pessimistischen » Einstellung zu den Geisteskrankheiten im Wege standen. Die Psychiatrie Amerikas sieht sich, seiner Meinung nach, heute einem ähnlichen Problem gegenüber. Trotz aller Veränderungen in der Psychiatrie habe das breite Publikum jetzt noch ähnliche Vorstellungen von den Geisteskrankheiten wie vor hundert Jahren.

Im ersten Teil des Buches bespricht DaIN die Anfänge der Psychiatrie in Amerika. Auf Rushs Bemühungen um die Behandlung der Kranken folgten die privaten Asyle, die das «moral management» zur hauptsächlichen Behandlungsmethode erhoben. Die Masse der Kranken wurde davon nicht berührt. Der zweite Teil schildert, wie das moral management scheiterte, als man versuchte, es in den neugegründeten großen staatlichen Anstalten anzuwenden. Mit zunehmendem Pessimismus wurden die hereditären prädisponierenden Hirnzustände immer mehr betont. An einer allumfassenden Theorie mangelte es jedoch, da die Anstaltsärzte Pragmatiker waren. Abgesehen von unzuverlässigen Statistiken wurde auch keine Forschung betrieben. Der dritte Teil berücksichtigt eingehend die Anstrengungen der Reformatoren, die Behandlung der Geisteskranken in den großen Anstalten auf eine humanitäre Basis zu bringen. 
Das Buch enthält viel wertvolles Material. Es ist zu begrüßen, daß auch nichtmedizinische Quellen herangezogen worden sind. Die ausführliche Berücksichtigung von außerhalb der Psychiatrie herrschenden Begriffen über Geisteskrankheit ist lobenswert. DaIN macht einen ehrlichen Versuch, die bisher nur glorifizierten Vorgänger in der amerikanischen Psychiatrie objektiv zu beurteilen.

Um so befremdender ist es, daß die almshouses und poorhouses, die doch fast alle Geisteskranken beherbergten, so wenig in Erscheinung treten und die medizinische Grundlage der damaligen Psychiatrie so wenig beachtet wird. Daß, wie A.Deutsch ${ }^{1}$ schreibt, für die meisten psychiatrischen Patienten die Zeit still stand, wird aus diesem Buch nicht klar ersichtlich. So kommen denn auch die Anliegen der Reformatoren eher überraschend, nachdem anfangs die Privatasyle im Buche die Hauptrolle gespielt haben.

Die Begriffe, die der Titel versprach, werden nicht oder nur ungenügend herausgestellt. Das mag daran liegen, daß «kein amerikanischer Psychiater eine klare psychologische Theorie hatte». Aber es gab ja auch nichtpsychologische Theorien. Denn auch das moral management war ja nicht das Resultat einer psychologischen Theorie, sondern ein Produkt der Aufklärung auf moralischer Grundlage ${ }^{2}$.

Niemand wird bestreiten, daß die «öffentliche Meinung wichtig ist, um eine Behandlung der Geisteskranken zu garantieren; und daß die Einstellung (zur Behandlung der Geisteskranken) der politischen und finanziellen Machthaber ausschlaggebend ist», wie DAIN abschließend sagt. Diese öffentliche Meinung und Überzeugung hat jedoch nicht unbedingt eine psychologische Theorie zur Grundlage.

Ich glaube nicht, daß die heutige Problematik der amerikanischen Psychiatrie mit den Schwierigkeiten der Behandlungsversuche mit moral management am Anfang des 19. Jahrhunderts vergleichbar ist. Moderne Milieutherapie und Psychotherapie sind nicht moral management in neuem Gewande. Ganz unvergleichbar ist das damalige Krankengut mit dem heutigen. Damals war Pessimismus im Hinblick auf die Prognose, besonders mit dem damaligen Wissen und Können, alles andere als überspitzt oder unnötig, sondern eher wissenschaftlich. Auf alle Fälle war es wirklichkeitsnäher als die unerfüllten und unerfüllbaren Versprechungen des moral management.

Was das Buch aufzeigt, ist, wieviel in der Psychiatrie ohne klare Begriffe geschrieben, behauptet und behandelt werden kann. An diesem Mangel leidet auch das Buch.

Otto M. Marx

9. M. Mrchler, Die Klumpfußlehre der Hippokratiker. Eine Untersuchung von De articulis cap. 62. Mit Übersetzung des Textes und des galenischen Kom-

1 A. Deutsch, The Mentally Ill in America, Doubleday, New York 1937.

2 E.H.Ackerknecht, Kurze Geschichte der Psychiatrie, Enke, Stuttgart 1957. 